\title{
Parasitismo em tambatinga (Colossoma macropomum x Piaractus brachypomus, Characidae) cultivados na Amazônia, Brasil
}

\author{
Márcia Kelly Reis DIAS ${ }^{1}$, Ligia Rigôr NEVES ${ }^{2}$, Renata das Graças Barbosa MARINHO ${ }^{1}$, \\ Douglas Anadias PINHEIRO ${ }^{3}$, Marcos TAVARES-DIAS , $^{1 *}$ \\ 1 Universidade Federal do Amapá (UNIFAP), Programa de Pós-Graduação em Biodiversidade Tropical, Rodovia Juscelino Kubitschek, km 5, 2600, Universidade, 68903-419, Macapá, AP, Brasil. \\ 2 Embrapa Amapá, Laboratório de Sanidade de Organismos Aquáticos, Rodovia Juscelino Kubitschek, km 5, 2600, Universidade, 68903-419, Macapá, AP, Brasil. \\ ${ }^{3}$ Universidade Estadual de Maringá (UEM), Programa de Pós-Graduação em Zootecnia (PPZ), Avenida Colombo, 5.790, Campus Universitário, 87020-900, Maringá, PR, Brasil. \\ * Autor correspondente: marcos.tavares@embrapa.br
}

\section{RESUMO}

Os estudos sobre os parasitos e doenças parasitárias são de grande interesse para a piscicultura, uma vez que podem afetar o crescimento dos peixes. O objetivo deste estudo foi investigar a fauna parasitária e relação parasito-hospedeiro em Colossoma macropomum x Piaractus brachypomus (tambatinga) de 10 pisciculturas do estado do Amapá, Amazônia. Dos 503 peixes examinados, 63,1\% estavam parasitados e 49.299 .189 parasitos foram coletados, tais como Ichthyophthirius multifiliis, Piscinoodinium pillulare, Trichodina sp., Tetrahymena sp., Anacanthorus spathulatus, Linguadactyloides brinkmanni, Mymarothecium boegeri, Notozothecium janauachensis, Procamallanus (Spirocamallanus) inopinatus, Neoechinorhynchus buttnerae e Perulernaea gamitanae. Porém, a dominância foi de I. multifiliis, seguida de P. pillulare e monogenoideas, parasitos que apresentaram padrão de dispersão agregado juntamente com $P$. gamitanae. Houve correlação positiva do comprimento dos hospedeiros com a prevalência parasitária total, bem como do tamanho dos peixes com a abundância de $I$. multiliis, $P$. pillulare, monogenoideas e $P$. gamitanae, mas os níveis infecção náo influenciaram o fator de condição relativo dos hospedeiros. A ocorrência de ectoparasitos foi favorecida pelo manejo e pobre condição sanitária das pisciculturas, mas a presença de espécies de endoparasitos foi devido ao abastecimento dos viveiros com água provenientes de corpos de água naturais. Este foi primeiro relato de I. multiliis, P. pillulare, Trichodina sp., Tetrahymena sp., A. spathulatus, N. janauachensis, N. buttnerae e P. (S.) inopinatus para tambatinga no Brasil.

PALAVRAS-CHAVE: Agregação, cultivo, parasitos, peixe, sanidade.

\section{Parasitism in tambatinga (Colossoma macropomum x Piaractus brachypomus, Characidae) farmed in the Amazon, Brazil}

\begin{abstract}
The studies on parasites and parasitic diseases are of great interest to fish farms, because both can affect the performance of the fish. The aim of study was to investigate the parasitic fauna and parasite-host relationship in Colossoma macropomum $\mathrm{x}$ Piaractus brachypomus (tambatinga) of 10 fish farms from the Amapá State, in the Amazon. Of the 503 fish examined 63.1\% were parasitized, and a total of 49,299,189 parasites were collected, including Ichthyophthirius multifliis, Piscinoodinium pillulare, Trichodina sp., Tetrahymena sp., Anacanthorus spathulatus, Linguadactyloides brinkmanni, Mymarothecium boegeri, Notozothecium janauachensis, Procamallanus (Spirocamallanus) inopinatus, Neoechinorhynchus buttnerae and Perulernaea gamitanae. Ichthyophthirius multifiliis was the dominant species, followed by P. pillulare and monogenoideans; these two parasites and $P$. gamitanae showed an aggregated dispersion pattern. We found a positive correlation between host length and the overall parasitic prevalence, as well as between fish size and the abundance of I. multiliis, P. pillulare, monogenoideans and $P$. gamitanae, but infection levels did not affect the relative condition factor of hosts. Management, low environmental quality and poor sanitary conditions of the fish farms favored the occurrence of ectoparasites, but the presence of endoparasites was due to supplying the ponds directly with water of natural water bodies. This is first report of $I$. multiliis, P. pillulare, Trichodina sp., Tetrahymena sp., A. spathulatus, $N$. janauachensis, $N$. buttnerae and $P$. (S.) inopinatus for tambatinga in Brazil.
\end{abstract}

KEYWORDS: Aggregation, culture, parasites, fish, sanity. 


\section{INTRODUÇÃO}

A aquicultura é o setor produtivo que mais cresce no mundo, em torno de $6,5 \%$ ao ano (Leung e Beates 2012). No Brasil, a aquicultura teve incremento de 31,1\% em 2011 (MPA 2013), principalmente influenciada pela produção da piscicultura. Apesar da rica diversidade de espécies nativas brasileiras e domínio da reprodução de algumas dessas espécies de importância zootécnica, o cultivo de peixes híbridos tem espaço garantido nas pisciculturas de diversas regióes, influenciado por fatores zootécnicos mais favoráveis para a criação intensiva (Pinheiro et al. 1991; Hashimoto et al. 2012). Entre esses peixes estão o híbrido produzido a partir do cruzamento de Colossoma macropomum (tambaqui) com Piaractus brachypomus (pirapitinga), conhecido popularmente como tambatinga, que tem superioridade em relação às suas espécies parentais quanto a produtividade (Hashimoto et al. 2012). Pois esse híbrido apresenta crescimento rápido, rusticidade, tolerância às variaçóes de temperatura e níveis de oxigênio (Silva-Acuña e Guevara 2002; Dias et al. 2012; Hashimoto et al. 2012). Além disso, como tem melhor rendimento de carcaça despertou grande interesse das indústrias de pescado. Consequentemente, a produção desse peixe híbrido vem crescendo, pois foi de 4.915,6 toneladas em 2010 e 14.326,4 toneladas em 2011, representando aumento de mais de 190\% (MPA 2013).

No estado do Amapá, a tambatinga é o segundo peixe mais cultivado, depois do tambaqui, mas seu manejo inadequado ocorre em boa parte das pisciculturas, devido a pouca experiência dos piscicultores e falta de assistência técnica, levando ao baixo crescimento dos peixes e a perdas econômicas na produção (Tavares-Dias 2011; Silva et al. 2013).

Países tropicais sofrem proporcionalmente as maiores perdas na aquicultura devido ao rápido surgimento de doenças causadas por parasitos, quando em situaçóes sanitárias precárias. Isso proporciona um menor tempo para mitigar as perdas se comparados aos países de clima temperado (Leung e Beates 2012). No Brasil, padróes epidemiológicos das doenças parasitárias em peixes cultivados são ainda desconhecidos, pois há poucos estudos sobre parasitoses, incluindo de tambatinga. $\mathrm{Na}$ Venezuela, Centeno et al. (2004) registraram infecçóes por Anacanthorus spatulatus (Monogenoidea), Trichodina sp., Epistylis sp. (Protozoa), Myxobolus sp. (Myxosporea) e Ergasilus sp. (Crustacea) para esse híbrido cultivado. No Brasil, Cohen e Kohn (2009) relataram a ocorrência de Mymarothecium boegeri (Monogenoidea) em dois indivíduos de tambatinga cultivados em Sobral (CE). Recentemente, Dias et al. (2012) registraram pela primeira vez a ocorrência de Linguadactyloides brinkmanni em tambatingas cultivados em Macapá, estado do Amapá. O objetivo deste estudo foi investigar a fauna parasitária e relação parasito- hospedeiro em híbridos tambatinga de 10 pisciculturas do estado do Amapá, região da Amazônia brasileira.

\section{MATERIAL E MÉTODOS}

No período de setembro de 2009 a abril de 2011, 503 espécimes de tambatinga $C$. macropomum x P. brachypomus (Characidae), sendo 208 alevinos e 295 peixes de engorda, foram coletados para análise parasitológica em 10 pisciculturas do município de Macapá, estado do Amapá. Essas pisciculturas apresentavam características e manejo diferenciados. Durante o período desse estudo, também foi acompanhado a mortalidade de peixes em cada piscicultura.

De cada peixe necropsiado foram examinados a boca, opérculos, brânquias e trato digestório. As brânquias foram removidas, fixadas em formol $5 \%$ e analisadas com auxílio de estereomicroscópio (SMZ 800N, Nikon, Tóquio, Japão) e microscópio (Eclipse E100, Nikon, Tóquio, Japão). O trato digestório removido foi examinado imediatamente em estereomicroscópio e os endoparasitos coletados e fixados. A metodologia empregada para coleta, fixação e preparação dos parasitos para identificação seguiu recomendaçóes prévias (Eiras et al. 2006; Thatcher 2006). A quantificação dos protozoários e metazoários foi de acordo com Tavares-Dias et al. $(2001 \mathrm{a}, \mathrm{b})$

Os descritores parasitários usados foram os recomendados por Rohde et al. (1995) e Bush et al. (1997). Foi usado o índice de dispersão (ID) para detectar o padrão de distribuiçẫo (agregado, uniforme ou aleatório) de cada infracomunidade de parasito (Rózsa et al. 2000) com prevalência $\geq 10 \%$. A significância do ID, para cada infracomunidade de parasito, foi testada pelo estatístico $d$ (Ludwig e Reynolds 1988). O índice de discrepância (D), uma estimativa da disparidade existente entre a distribuição observada e uma distribuição uniforme do número de indivíduos das infracomunidades parasitárias foi calculado usando o software Quantitative Parasitology 3.0 (Rózsa et al. 2000).

Os dados de peso $(\mathrm{g})$ e comprimento total $(\mathrm{cm})$ foram usados para calcular a equação da relação peso-comprimento e fator de condição relativo $(\mathrm{Kn})$ de peixes parasitados e náo parasitados. $\mathrm{Kn}=\mathrm{P}_{\mathrm{o}} / \mathrm{P}_{\mathrm{e}}$, onde $\mathrm{P}_{\mathrm{o}}=$ peso observado e $\mathrm{P}_{\mathrm{e}}=$ peso esperado obtido da relaçáo peso-comprimento (Le-Cren 1951). A diferença nos valores do fator de condição relativo, entre peixes parasitados e não parasitados, foi comparada pelo teste de Mann-Whitney $(U)$. O coeficiente de Pearson (r) foi usado para determinar possíveis correlaçóes da abundância das infracomunidades de parasitos com comprimento, peso e Kn dos hospedeiros, bem como entre a abundância de monogenoideas com a abundância de $I$. multifiliis e $P$. pillulare (Zar 1999). A existência de correlação entre o comprimento total dos hospedeiros e a prevalência de infecção parasitária total foi testada usando o coeficiente de Pearson (r), com prévia transformação angular dos dados de prevalência (arco seno) e separação das amostras em 9 classes de comprimento (3,0-9,5 
$\mathrm{cm} ; 9,7-14,5 \mathrm{~cm} ; 15,0-19,5 \mathrm{~cm} ; 20,0-25,6 \mathrm{~cm} ; 26,0-29,8 \mathrm{~cm}$; $30,0-34,5 \mathrm{~cm} ; 35,0-39,8 \mathrm{~cm} ; 40,0-44,9 \mathrm{~cm} ; 45,0-49,0 \mathrm{~cm})$.

Em cada piscicultura, durante as coletas dos peixes, foram determinados o $\mathrm{pH}$ usando $\mathrm{pHmetro}$ digital $(\mathrm{pH}$ 100, YSI, Brannum Lane, OH, USA), temperatura e níveis de oxigênio dissolvido usando oxímetro digital (DO-200, YSI, Brannum Lane, OH, USA). Para comparação desses parâmetros entre pisciculturas foi usada análise de variância (ANOVA), seguida pelo teste de Dunn, quando houve diferenças significativas $(\mathrm{P}<0,05)$.

\section{RESULTADOS}

Nos viveiros de cultivo das 10 pisciculturas os valores médios da temperatura e oxigênio dissolvido foram similares, porém esses níveis de oxigênio estiveram abaixo de $3 \mathrm{mg} \mathrm{L}^{-1}$ na maioria das propriedades. Os valores médios de $\mathrm{pH}$ mostraram diferenças significativas $(\mathrm{P}<0,05)$ somente em viveiros de duas pisciculturas (Figura 1).

Os peixes foram cultivados em diferentes densidades de estocagem e apresentavam tamanhos variados, devido as diferentes fases de cultivo (alevinagem e recria). Assim, a prevalência parasitária total variou entre as pisciculturas investigadas (Tabela 1).

Durante o período deste estudo, observou-se a mortalidade de cinco mil alevinos $( \pm 5 \mathrm{~cm})$ da piscicultura 4 , mas nenhum peixe examinado estava parasitado. A mortalidade ocorreu após transporte inadequado durante horário (entre 12 e 14 horas) de temperaturas mais elevadas do dia e sem o uso de oxigênio.

Nas 10 pisciculturas investigadas, os peixes estavam parasitados por Ichthyophthirius multifliiis Fouquet, 1876; Tetrahymena sp.; Piscinoodinium pillulare (Schäperclaus, 1954) Lom, 1981; Trichodina sp.; Perulernaea gamitanae Thatcher e Paredes, 1985, Anacanthorus spathulatus Kritsky, Thatcher e Kayton 1979; Linguadactyloides brinkmanni Thatcher e Kritsky, 1983; Mymarothecium boegeri Cohen e Kohn 2005; Notozothecium janauachensis Belmont-Jégu, Domingues e Martins 2004; Procamallanus (Spirocamallanus) inopinatus Travassos, Artigas e Pereira, 1928 e Neoechinorhynchus buttnerae Golvan, 1956. As infecçôes por I. multifiliis foram similares em peixes de $70 \%$ das pisciculturas, enquanto parasitismo por $P$. pillulare não ocorreu em duas pisciculturas (Tabela 2). Baixa prevalência e intensidade de Trichodina sp. (prevalência $=5,2 \%$ e intensidade média $=1.220 \pm 2.009$ parasitos por peixe) e Tetrahymena sp. ( $\mathrm{P}=7,8 \%$ e IM $=505 \pm$ 441 parasitos por peixe) ocorreram somente na piscicultura 5 .

A prevalência de espécies de monogenoideas foi similar nas brânquias de híbridos tambatinga de $80 \%$ das pisciculturas, mas somente A. spathulatus parasitou peixes de todas as pisciculturas. Porém, $M$. boegeri ocorreu em peixes de $60,0 \%$ das pisciculturas, L. brinkmanni e N. janauachensis em 30,0\% das pisciculturas (Tabela 3).

Procamallanus $(S$.) inopinatus ocorreu no intestino de peixes de somente três pisciculturas e em baixa prevalência, intensidade média e abundância (Tabela 3). Porém, acantocéfalo $N$. buttnerae foi encontrado no intestino de
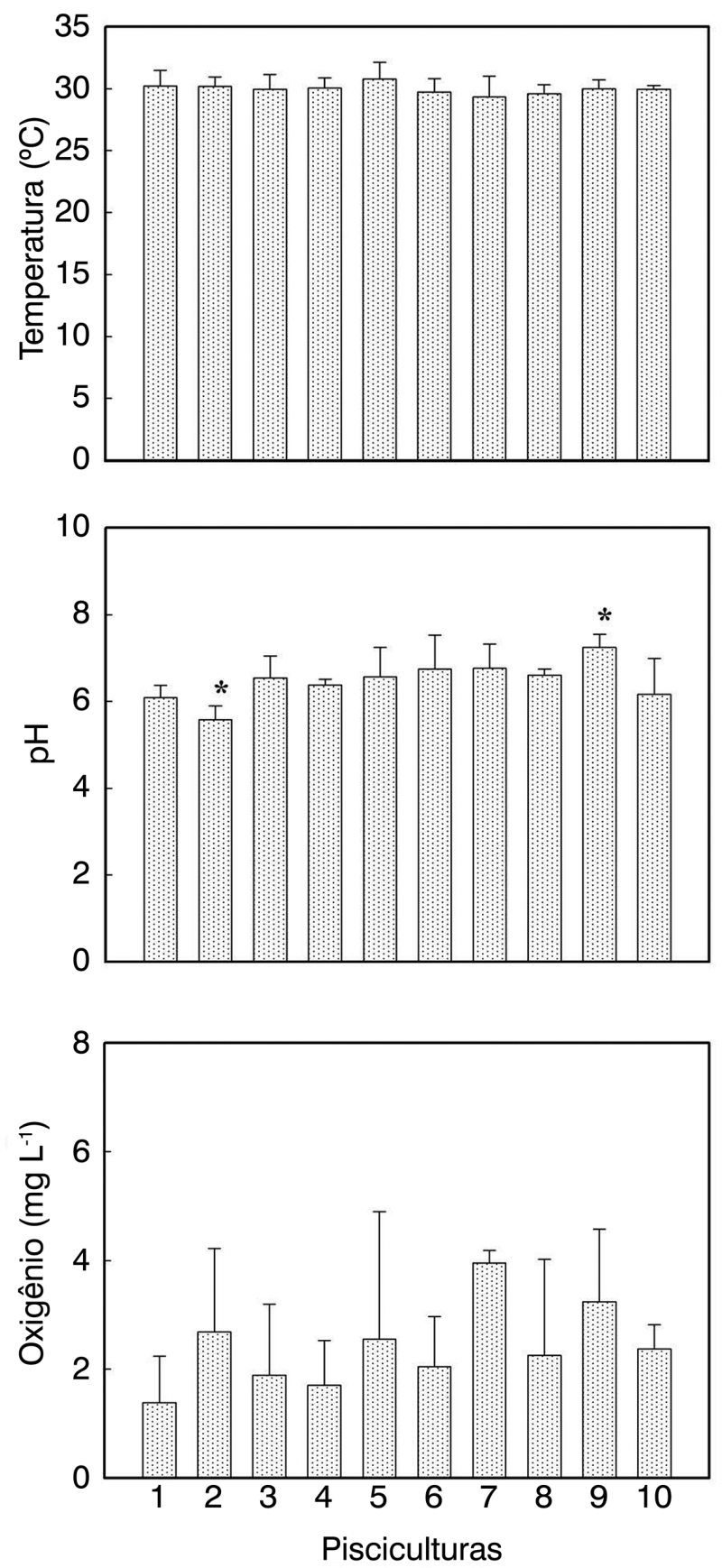

Figura 1. Parâmetros de qualidade da água nos viveiros de tambatinga em 10 pisciculturas do estado do Amapá, Amazônia brasileira. Média \pm desvio padrão. * Indica diferenças pelo teste de Dunn $(\mathrm{P}<0,05)$. 
Tabela 1. Peso, comprimento médio ( \pm desvio padrão) e número (N) de híbridos tambatinga coletados em pisciculturas do estado do Amapá, Amazônia brasileira. P: Prevalência parasitária total.

\begin{tabular}{|c|c|c|c|c|c|c|}
\hline Pisciculturas & Coordenadas geográficas & $\mathrm{N}$ & Peso $(\mathrm{g})$ & Comprimento $(\mathrm{cm})$ & Densidade (peixe $\mathrm{m}^{-2}$ ) & $\mathrm{P}(\%)$ \\
\hline 1 & $00^{\circ} 08^{\prime} 42.3^{\prime \prime} \mathrm{N}, 051^{\circ} 06^{\prime} 04.2^{\prime \prime} \mathrm{W}$ & 58 & $687,4 \pm 459,23$ & $31,6 \pm 7,86$ & 2,5 & 86,0 \\
\hline 2 & $0^{\circ} 0^{\prime} 21.7^{\prime \prime} \mathrm{S}, 051^{\circ} 07^{\prime} 23.1^{\prime \prime} \mathrm{W}$ & 60 & $461,4 \pm 340,4$ & $27,8 \pm 8,4$ & 2,0 & 58,3 \\
\hline 3 & $0^{\circ} 00^{\prime} 13.5^{\prime \prime} \mathrm{S}, 051^{\circ} 06^{\prime} 12.8^{\prime \prime} \mathrm{W}$ & 66 & $986,7 \pm 597,75$ & $34,3 \pm 11,45$ & 2,8 & 59,1 \\
\hline 4 & $0^{\circ} 05^{\prime} 02^{\prime \prime} \mathrm{N}, 051^{\circ} 02^{\prime} 44.2^{\prime \prime} \mathrm{W}$ & 33 & $542,8 \pm 372,24$ & $28,5 \pm 8,44$ & 0,6 & 100,0 \\
\hline 5 & $00^{\circ} 01^{\prime} 48.3^{\prime \prime} \mathrm{S}, 051^{\circ} 07^{\prime} 52.9^{\prime \prime} \mathrm{W}$ & 73 & $244 \pm 188,91$ & $17,5 \pm 7,64$ & 2,0 & 79,2 \\
\hline 6 & $0^{\circ} 04^{\prime} 15.4^{\prime \prime} \mathrm{N}, 051^{\circ} 02^{\prime} 16.9^{\prime \prime} \mathrm{W}$ & 70 & $470,9 \pm 235,27$ & $29,3 \pm 6,13$ & 2,8 & 69,1 \\
\hline 7 & $0^{\circ} 02^{\prime} 31.4^{\prime \prime S}, 051^{\circ} 07^{\prime} 34.4^{\prime \prime} \mathrm{W}$ & 58 & $898 \pm 630,79$ & $32,6 \pm 11,93$ & 1,2 & 86,2 \\
\hline 8 & $00^{\circ} 00^{\prime} 13.2^{\prime \prime} \mathrm{S}, 051^{\circ} 06^{\prime} 10.9^{\prime \prime} \mathrm{W}$ & 30 & $62,8 \pm 66,13$ & $11,31 \pm 6,24$ & 0,8 & 13,3 \\
\hline 9 & $0^{\circ} 00^{\prime} 04.5^{\prime \prime} \mathrm{N}, 051^{\circ} 05^{\prime} 52.1^{\prime \prime} \mathrm{W}$ & 35 & $334,3 \pm 237,2$ & $20,2 \pm 6,24$ & 1,5 & 77,1 \\
\hline 10 & $0^{\circ} 06^{\prime} 32.64^{\prime \prime} \mathrm{N}, 051^{\circ} 03^{\prime} 40.66^{\prime \prime} \mathrm{W}$ & 20 & $491,95 \pm 493$ & $25,2 \pm 13,32$ & 1,2 & 50,0 \\
\hline
\end{tabular}

Tabela 2. Protozoários parasitos das brânquias de híbrido tambatinga em pisciculturas do estado do Amapá, Amazônia brasileira. PE: Peixes examinados, PP: Peixes parasitados, IM: Intensidade média, AM: Abundância média, ATP: Abundância total de parasitos.

\begin{tabular}{|c|c|c|c|c|c|c|c|c|c|c|}
\hline \multirow[b]{2}{*}{ Pisciculturas } & \multicolumn{5}{|c|}{ Ichthyophthirius multifiliis } & \multicolumn{5}{|c|}{ Piscinoodinium pillulare } \\
\hline & $\mathrm{PE} / \mathrm{PP}$ & $\mathrm{P}(\%)$ & IM & AM & ATP & $\mathrm{PE} / \mathrm{PP}$ & $\mathrm{P}(\%)$ & IM & $A M$ & ATP \\
\hline 1 & $57 / 49$ & 86,0 & 88.026 & 75.671 & 431.3267 & $57 / 23$ & 40,4 & 41.872 & 16.896 & 963.047 \\
\hline 2 & $60 / 32$ & 46,7 & 59.737 & 31.860 & 1.911 .598 & $60 / 28$ & 46,7 & 13.639 & 7.274 & 436.456 \\
\hline 3 & $66 / 39$ & 59,1 & 323.119 & 190.934 & 12.601 .657 & $66 / 10$ & 15,2 & 247.162 & 37.448 & 2.471.617 \\
\hline 4 & $32 / 21$ & 65,6 & 17.969 & 11.792 & 377.351 & $32 / 0$ & 0 & 0 & 0 & 0 \\
\hline 5 & $77 / 56$ & 72,7 & 140.225 & 101.982 & 7.852 .587 & $77 / 28$ & 36,4 & 13.596 & 4.944 & 380.696 \\
\hline 6 & $68 / 43$ & 63,2 & 125.697 & 79.485 & 5.404 .991 & $68 / 29$ & 42,6 & 49.560 & 21.136 & 1.437 .251 \\
\hline 7 & $58 / 26$ & 44,8 & 326.783 & 146.489 & 8.496 .369 & $58 / 10$ & 17,2 & 4.551 & 785 & 45.513 \\
\hline 8 & $30 / 4$ & 13,3 & 92.632 & 12.351 & 370.526 & $30 / 4$ & 13,3 & 16.062 & 2.142 & 64.248 \\
\hline 9 & $35 / 14$ & 40,0 & 109.512 & 43.804 & 1.533 .168 & $35 / 5$ & 14,3 & 68.446 & 9.778 & 342.230 \\
\hline 10 & $20 / 9$ & 45,0 & 28.708 & 12.919 & 258.372 & $20 / 0$ & 0 & 0 & 0 & 0 \\
\hline
\end{tabular}

Tabela 3. Helmintos parasitos de híbridos tambatinga em pisciculturas do estado do Amapá, Amazônia brasileira. PE: Peixes examinados, PP: Peixes parasitados, IM: Intensidade média, AM: Abundância média, ATP: Abundância total de parasitos.

\begin{tabular}{|c|c|c|c|c|c|c|c|c|c|c|}
\hline \multirow[b]{2}{*}{ Pisciculturas } & \multicolumn{5}{|c|}{ Monogenoidea } & \multicolumn{5}{|c|}{ Procamallanus (S.) inopinatus } \\
\hline & $\mathrm{PE} / \mathrm{PP}$ & $\mathrm{P}(\%)$ & IM & AM & ATP & $\mathrm{PE} / \mathrm{PP}$ & $\mathrm{P}(\%)$ & IM & AM & ATP \\
\hline 1 & $57 / 49$ & 86,0 & 77 & 66 & 3.777 & $57 / 0$ & 0 & 0 & 0 & 0 \\
\hline 2 & $60 / 25$ & 41,7 & 57 & 33 & 1.984 & $60 / 0$ & 0 & 0 & 0 & 0 \\
\hline 3 & $66 / 39$ & 59,1 & 194 & 115 & 7.562 & $66 / 0$ & 0 & 0 & 0 & 0 \\
\hline 4 & $32 / 21$ & 65,6 & 129 & 85 & 2.716 & $32 / 0$ & 0 & 0 & 0 & 0 \\
\hline 5 & $77 / 61$ & 79,2 & 108 & 86 & 6.605 & $77 / 5$ & 6,5 & 2 & 0,1 & 9 \\
\hline 6 & $68 / 47$ & 69,1 & 129 & 89 & 6.079 & $68 / 0$ & 0 & 0 & 0 & 0 \\
\hline 7 & $58 / 31$ & 53,4 & 90 & 48 & 2.782 & $58 / 1$ & 1,7 & 1 & 0,02 & 1 \\
\hline 8 & $30 / 4$ & 13,3 & 5 & 0,4 & 14 & $30 / 0$ & 0 & 0 & 0 & 0 \\
\hline 9 & $35 / 22$ & 62,9 & 33 & 20 & 691 & $35 / 1$ & 2,9 & 4 & 0,1 & 4 \\
\hline 10 & $20 / 10$ & 50,0 & 14 & 7 & 139 & $20 / 0$ & 0 & 0 & 0 & 0 \\
\hline
\end{tabular}


peixes somente da piscicultura 4, com prevalência de 43,8\% e intensidade média de $44 \pm 39$ parasitos por peixe.

Perulernaea gamitanae foi coletado somente nas brânquias e boca de peixes das pisciculturas 5 e 7 . Em peixes da piscicultura 5 os parasitos foram observados na boca ( $\mathrm{P}=13,2 \%$ e $\mathrm{IM}=3 \pm 2$ parasitos por peixe) e brânquias ( $\mathrm{P}=6,5 \%$ e $\mathrm{IM}=2 \pm 1$ parasitos/peixe) dos peixes, mas em peixes da piscicultura 7 o parasitismo foi maior, tanto na boca $(\mathrm{P}=53,4 \%$ e $\mathrm{IM}=16 \pm 12$ parasitos por peixe $)$ como nas brânquias ( $\mathrm{P}=34,5 \%$ e IM $=10 \pm 6$ parasitos por peixe). Houve dominância de I. multifliiis, seguido por P. pillulare e espécies de monogenoideas (Tabela 4) e padrão de distribuição agregado de parasitos, mas o índice de discrepância (D) indica uma maior agregação de $P$. pillulare e P. gamitanae (Tabela 5).
A relação peso-comprimento foi alométrica negativa (y $\left.=0,0657 \mathrm{x}^{2,628} ; \mathrm{r}^{2}=0,841\right)$ para peixes não parasitados $(\mathrm{y}=$ $\left.0,0294 \mathrm{x}^{2.8555} ; \mathrm{r}^{2}=0,975\right)$ e parasitados $\left(\mathrm{y}=0.0664 \mathrm{x}^{2.6251} ; \mathrm{r}^{2}=\right.$ $0,841)$, indicando maior incremento em massa corporal que em tamanho. Não houve diferença significativa $(U=22013.5$; $\mathrm{P}=0,233)$ entre o fator de condição relativo $(\mathrm{Kn})$ de peixes parasitados $(K n=1,011 \pm 0,158)$ e não parasitados $(K n=$ $1,071 \pm 0,561)$.

A abundância de monogenoideas, I. multifiliis, $P$. pillulare e $P$. gamitanae mostrou correlaçáo positiva com o comprimento total, peso corporal e fator de condição relativo dos hospedeiros (Tabela 6). Foi observada correlaçáo positiva do comprimento dos hospedeiros com a prevalência parasitária total $(\mathrm{r}=0,571 ; \mathrm{P}=0,0001)$. A abundância de monogenoideas mostrou correlação positiva com a abundância de I. multifiliis $(\mathrm{r}=0,360 ; \mathrm{P}=0,0001)$ e $P$. pillulare $(\mathrm{r}=0,228 ; \mathrm{P}=0,0001)$.

Tabela 4. Índices parasitológicos em híbridos tambatinga cultivados no estado do Amapá, Amazônia brasileira. SI: Sítio de infecção, PE: Peixes examinados, PP: Peixes parasitados, IM: Intensidade média, DP: Desvio padrão, AM: Abundância média, ATP: Abundância total de parasitos, DR: dominância relativa.

\begin{tabular}{lccccccc}
\hline Parasitos & SI & PE/PP & P (\%) & IM \pm DP & AM & ATP & DR \\
\hline Ichthyophthirius multifilis & Brânquias & $503 / 293$ & 58,3 & $85.725,4 \pm 15.1828,8$ & 147.167 & $43.119 .886,0$ & 0,87459 \\
Piscinoodinium pillulare & Brânquias & $503 / 141$ & 28,0 & $12.208,9 \pm 43.006,2$ & 43.554 & $6.141 .058,0$ & 0,12456 \\
\hline Tetrahymena sp. & Brânquias & $503 / 6$ & 1,2 & $6,6 \pm 74,7$ & 555 & 3.332 & 0,00007 \\
Trichodina sp. & Brânquias & $503 / 4$ & 0,8 & $9,7 \pm 189,4$ & 1.220 & 1.220 & 0,00019 \\
Monogenoidea & Brânquias & $503 / 317$ & 63,0 & $64,3 \pm 97,6$ & 102 & 32.344 & 0,00066 \\
Procamallanus (S.) inopinatus & Intestino & $503 / 7$ & 1,4 & $0.03 \pm 0,23$ & 2 & 14 & - \\
Neoechinorhynchus buttnerae & Intestino & $503 / 14$ & 2,8 & $1,2 \pm 9,7$ & 44 & 622 & 0,00001 \\
Perulernaea gamitanae & Brânquias & $503 / 25$ & 5,0 & $0,4 \pm 2,3$ & 9 & 215 & - \\
Perulernaea gamitanae & Boca & $503 / 40$ & 8,0 & $0,99 \pm 4,82$ & 12 & 498 & 0,00001 \\
\hline
\end{tabular}

Tabela 5. Índice de dispersão (ID), estatístico de índice de discrepância (D) para híbridos tambatinga $(\mathrm{N}=503)$ de pisciculturas do estado do Amapá, Amazônia brasileira.

\begin{tabular}{lccc}
\hline Parasitos & ID & d & D \\
\hline Ichthyophthirius multifiliis & 16,812 & 98,25 & 0,595 \\
Piscinoodinium pillulare & 11,556 & 76,04 & 0,804 \\
Monogenoidea & 8,969 & 63,22 & 0,641 \\
Perulernaea gamitanae & 6,730 & 50,53 & 0,950 \\
\hline
\end{tabular}

Tabela 6. Coeficiente da correlação de Pearson ( $r$ ) entre a abundância de parasitos e os parâmetros corporais e fator de condição relativo $(\mathrm{Kn})$ de híbrido tambatinga ( $\mathrm{N}=503$ ) em pisciculturas do estado do Amapá, Amazônia brasileira. P: probabilidade.

\begin{tabular}{lcccccccc}
\hline \multirow{2}{*}{ Parasitos } & \multicolumn{2}{c}{ Comprimento } & & \multicolumn{2}{c}{ Peso corporal } & & \multicolumn{2}{c}{$\mathrm{Kn}$} \\
\cline { 2 - 3 } \cline { 8 - 9 } & $\mathrm{r}$ & $\mathrm{P}$ & & $\mathrm{r}$ & $\mathrm{P}$ & & $\mathrm{r}$ & $\mathrm{P}$ \\
\hline I. multifiliis & 0,3234 & 0,0001 & & 0,3189 & 0,0001 & & 0,2516 & 0,0001 \\
P. pillulare & 0,2383 & 0,0001 & & 0,3187 & 0,0001 & & 0,1847 & 0,0001 \\
Monogenoidea & 0,2310 & 0,0001 & & 0,1925 & 0,0001 & & 0,2278 & 0,0001 \\
\hline P. gamitanae & 0,2815 & 0,0001 & & 0,3685 & 0,0001 & & 0,1828 & 0,0001 \\
\hline
\end{tabular}

\section{DISCUSSÃO}

Em tambatinga cultivada no estado do Amapá houve dominância de ectoparasitos I. multifiliis, P. pillulare, monogenoideas e P. gamitanae. Porém, esse mesmo hospedeiro cultivado na Venezuela foi parasitado por A. spatulatus, Trichodina sp., Epistylis sp., Myxobolus sp. e Ergasilus sp (Centeno et al. 2004). Os peixes podem ser parasitados por diversas espécies de parasitos, os quais podem estar dispersos de forma diferente nos hospedeiros (Rohde et al. 1995; Moller 2006). A dispersão agregada de parasitos está relacionada principalmente a sua estratégia e reprodução direta, heterogeneidade dos peixes quanto à suscetibilidade aos parasitos e ao diferenciado sistema imunológico dos hospedeiros, de forma que esse padrão de agregaçáo estabiliza a dinâmica da relação parasito-hospedeiro, controlando a populaçấo hospedeira (Moller 2006).

Em tambatinga de diferentes pisciculturas foram encontrados elevados níveis de infecçóes por protozoários I. multifiliis e $P$. pillulare, além de baixo parasitismo por 
Trichodina sp. e Tetrahymena sp. Os níveis de infecção por tais parasitos são, em geral, influenciados principalmente pelo manejo e baixas condiçóes ambientais do cultivo (Banu e Khan 2004; Santos et al. 2013; Silva et al. 2013). A maioria das pisciculturas apresentou baixos níveis de oxigênio dissolvido na água e condição sanitária inadequada, o que favorece a prevalência de parasitos. Assim, houve correlação positiva da abundância de monogenoideas com a abundância de $I$. multifliis e P. pillulare, ambos parasitos oportunistas (TavaresDias et al. 2001b; Silva et al. 2013). Porém, em nenhuma das pisciculturas investigadas os peixes apresentaram sinais clínicos de doenças, comprovando a propalada rusticidade desse híbrido no cultivo intensivo (Silva-Acuña e Guevara 2002; Dias et al. 2012; Hashimoto et al. 2012).

Embora A. spathulatus, M. boegeri, N. janauachensis e L. brinkmanni tenham sido encontrados parasitando as brânquias dos peixes deste estudo, somente $A$. spathulatus ocorreu em hospedeiros de todas as pisciculturas, enquanto $M$. boegeri ocorreu em $60,0 \%$ das propriedades investigadas e L. brinkmanni e $N$. janauachensis apenas em 30,0\% delas. Porém, A. spathulatus foi o único monogenoidea parasitando as brânquias de tambatingas $(\mathrm{n}=120)$ cultivadas na Venezuela (Centeno $e t$ al. 2004), e a prevalência de monogenoideas foi similar ao do presente estudo, mas para esse mesmo peixe $(\mathrm{n}=$ 2) cultivo em Sobral (CE) foi relatado somente a ocorrência de M. boegeri (Cohen e Kohn 2009). Tais diferenças são, portanto, devido ao baixo número de peixes examinados em Sobral.

Anacanthorus spathulatus é um monogenoidea patogênico que pode causar redução na capacidade respiratória dos peixes hospedeiros (Morais et al. 2009; Santos et al. 2013; Silva et al. 2013) dependendo dos níveis de infecçâa, assim como $L$. brinkmanni, o qual provoca reação inflamatória severa e grave hiperplasia acompanhada por hemorragias (Centeno et al. 2004; Thatcher 2006). Portanto, são necessárias medidas de controle desses parasitos e melhorias sanitárias, principalmente quando os peixes apresentarem infecçóes múltiplas como ocorreram neste estudo. Como os monogenoideas são ectoparasitos de ciclo de vida direto, sua reproduçấo é favorecida pela elevada temperatura e densidade populacional dos peixes, bem como pelos baixos níveis de oxigênio na água dos viveiros (Banu e Khan 2004; Modu et al. 2012), principalmente em regiōes tropicais como a Amazônia onde as temperaturas são elevadas e constante durante todo o ano.

Perulernaea gamitanae ocorreu nas brânquias e boca dos peixes de apenas duas pisciculturas, mas o maior nível de parasitismo foi em peixes de uma das propriedades (piscicultura 7). Constatouse que houve a transmissão desses ectoparasitos para uma outra piscicultura (piscicultura 5) que adquiriu tambaquis infestados com esse lerneídeo amazônico que possui especificidade parasitária. Esses resultados demonstram a grande importância da quarentena e cuidados profiláticos, geralmente negligenciados por boa parte das pisciculturas da regiáo deste estudo (TavaresDias 2011; Silva et al. 2013), como também de outras regiôes brasileiras. Porém, os níveis moderados de parasitismo por $P$. gamitanae em peixes deste estudo foram maiores que aqueles descritos para C. macropomum de pisciculturas de Rondônia (Godoi et al. 2012). Portanto, devido a todos esses resultados e o constante transporte de alevinos, entre as pisciculturas de produção de alevinos e recria no estado do Amapá, devem ser tomados cuidados sanitários adequados para evitar a transmissão de P. gamitanae para outras propriedades. Elevado parasitismo por esses lerneídeos causaram epizootia em alevinos de $C$. macropomum cultivados na Amazônia peruana, levando a perdas econômicas (Delgado et al. 2011), principalmente em peixes juvenis. Embora peixes deste estudo tenham apresentado elevada agregação parasitária, não ocorreu mortalidade pois eram peixes maiores e com boas condiçóes corporais.

Procamallanus (S.) inopinatus ocorreu somente em tambatinga de três pisciculturas e em baixo nível de infecção, indicando baixa presença de hospedeiros intermediários no ambiente, como esperado em cultivo. Além disso, somente em peixes de uma das pisciculturas foi observado moderado nível de infecção por $N$. buttnerae (prevalência de 43,7\% e intensidade média de 44,4 parasitos por peixe) quando comparado a alevinos de C. macropomum cultivados no estado do Amazonas, os quais apresentaram $100 \%$ de prevalência e intensidade média de 30 a 406 parasitos, que causou mortalidade devido a obstrução intestinal (Malta et al. 2001). Esse mesmo acantocéfalo também foi encontrado parasitando híbridos tambacu (prevalência $=12,5 \%$ e intensidade média = 18,5 parasitos por peixe) (Silva et al. 2013) da mesma piscicultura do presente estudo. Neoechinorhynchus buttnerae ainda náo havia sido descrito infectando tambatinga, pois este é o primeiro registro. As espécies de Neoechinorhynchus são comuns em populações naturais onde há presença de hospedeiros intermediários. O ciclo de vida desses endohelmintos é complexo e tem uma espécie de ostracoide como hospedeiro intermediário primário (Martínez-Aquino et al. 2009). Larvas encontradas encistadas indicam que esse peixe pode ser hospedeiro intermediário para aquele parasito.

Apesar do parasitismo de híbridos tambatinga cultivados no estado do Amapá, o fator de condição relativo não foi influenciado, pois a maior abundância de I. multifiliis, $P$. pillulare, monogenoideas e $P$. gamitanae foi observada em hospedeiros maiores e com melhores condiçôes corporais, assim os parasitos não foram patogênicos. Resultados similares foram relatados para C. macropomum cultivados quando parasitados por esses mesmos parasitos (Godoi et al. 2012; Santos et al. 2013). Porém, Pojmanska (1994) observaram que geralmente a abundância de espécies de protozoários é maior em peixes menores e os crustáceos ectoparasitos infectam peixes maiores, enquanto os monogenoideas parasitam 
peixes de todos os tamanhos, dependendo da espécie de dactilogirídeos.

\section{CONCLUSÕES}

Os resultados mostram que a prevalência de parasitos é influenciada pelo diferenciado manejo das pisciculturas no que se refere, principalmente, à densidade de estocagem dos peixes e quantidade da alimentação, pois em algumas propriedades esses permanecem por certo período sem qualquer alimentação balanceada (ração) ou são alimentados com ração estragada ou sem qualquer balanceamento. Portanto, a presença de ectoparasitos com ciclo de vida direto é favorecida pela pobre condição sanitária do ambiente de cultivo dos peixes. Embora a maioria das pisciculturas use corpos de água naturais (rio, igarapé e várzea) para abastecimento dos viveiros, a diversidade de endohelmintos é baixa, pois são parasitos com ciclo de vida complexo, dependente de hospedeiros intermediários contendo formas infectantes, que quando presentes no ambiente de cultivo podem ocorrer geralmente em baixa abundância. Esses dados parasitários e o padrão de distribuição indicam a necessidade de adoção de medidas profiláticas adequadas nas pisciculturas estudadas, para evitar perdas econômicas futuras devido a epizootias. Este é o primeiro relato desses protozoários, além de $A$. spathulatus, $N$. buttnerae, $N$. janauachensis e $P$. (S.) inopinatus para tambatinga no Brasil, e o terceiro registro de $M$. boegeri para esse hospedeiro.

\section{AGRADECIMENTOS}

Este trabalho foi desenvolvido de acordo com os princípios adotados pelo Colégio Brasileiro de Experimentação Animal (COBEA). Os autores são agradecem ao Conselho Nacional de Desenvolvimento Científico e Tecnológico (CNPq) pelo suporte financeiro (Proc. 578159/2008-2) e bolsa PQ concedida a Tavares-Dias, M.

\section{BIBLIOGRAFIA CITADA}

Banu, A.N.H.; Khan, M.H. 2004. Water quality, stocking and parasites of freshwater fish in four selected areas of Bangladesh. Pakistan Journal of Biological Sciences, 7: 436-440.

Bush, A.O.; Lafferty, K.D.; Lotz, J.M.; Shostak, A.W. 1997. Parasitology meets ecology on its own terms: Margolis et al. revisited. Journal of Parasitology, 83: 575- 583.

Centeno, L.; Silva-Acuña, A.; Silva-Acuña, R.; Pérez J.L. 2004. Fauna ectoparasitaria associada a Colossoma macropomum y al híbrido de C. macropomum x Piaractus brachypomus, cultivados em el Estado Delta Amacuro, Venezuela. Bioagro, 16: 121-126.

Cohen, S.C.; Kohn, A. 2009. On Dactylogyridae (Monogenea) of four species of characid fishes from Brazil. Check List, 5: 351-356.

Delgado, P.M.; Delgado, J.P.M.; Rosa, J.V.A.; Orbe, I. 2011. Infestación masiva por Perulernaea gamitanae (Crustacea: Cyclopoida: Lernaeidae) en juveniles de gamitana, cultivados en la Amazonia peruana. Veterinaria México, 42: 59-64.
Dias, M.K.R.; Tavares-Dias M.; Marchiori N. 2012. First report of Linguadactyloides brinkmanni (Monogenoidea: Linguadactyloidinae) on hybrids of Colossoma macropomum $\mathrm{x}$ Piaractus mesopotamicus (characidae) from South America. Brazilian Journal of Aquatic Science and Technology, 16: 61-64.

Eiras, J.C.; Takemoto, R.M.; Pavanelli, G.C. 2006. Métodos de estudo e técnicas laboratoriais em parasitologia de peixes. 2a Ed. Eduem, Maringá, 199p.

Godoi, M.M.I.M.; Engracia, V.; Lizama, M.L.A.P.; Takemoto, R.M. 2012. Parasite-host relationship between the tambaqui (Colossoma macropomum Cuvier 1818) and ectoparasites, collected from fish farms in the city of Rolim de Moura, State of Rondônia, Western Amazon, Brazil. Acta Amazonica, 42: 515-524.

Hashimoto, D.T.; Senhorini, J.A.; Foresti F.; Porto-Foresti, F. 2012. Interspecific fish hybrids in Brazil: management of genetic resources for sustainable use. Reviews in Aquaculture, 4: 108-118.

Le-Cren, E.D. 1951. The length-weight relationship and seasonal cycle in gonadal weight and condition in the perch (Perca Aluviatilis). Journal of Animal Ecology, 20: 201-219.

Leung, T.L.F.; Bates, A.E. 2012. More rapid and severe disease outbreaks for aquaculture at the tropics: implications for food security. Journal of Applied Ecology, 50: 215-222.

Ludwig, J.A.; Reynolds, J.F. 1988. Statistical ecology: A primer on methods and computing. Wiley-Interscience Pub, New York, 337p.

Malta, J.C.O.; Gomes, A.L.S.; Andrade, S.M.S. 2001. Infestação maciça por acantocéfalos Neochinorhynchus buttnerae Golvan, 1956, (Eoacanthocephala: Neoechinorhynchidae) em tambaquis jovens, Colossoma macropomum (Cuvier, 1818) cultivados na Amazônia central. Acta Amazonica, 31: 133-143.

Martínez-Aquino, A.; Reyna-Fabián, M.E.; Rosas-Valdez, R.; RazoMendivil, U.; Pérez-Ponce De León, G.; García-Varela, M. 2009. Detecting a complex of cryptic species within Neoechinorhynchus golvani (Acanthocephala: Neoechinorhynchidae) inferred from ITSs and LSU rDNA gene sequences. The Journal of Parasitology, 5: 1040-1047.

Ministério da Pesca E Aquicultura - MPA. 2013. Boletim estatístico da pesca e aquicultura 2011. Brasília, DF, 60p.

Modu, B.M.; Siful, M.; Kasin, M.K.; Hassan M.; Shahorom-Harrison, F.M. 2012. Effects of water quality and monogenean parasite in gills of freshwater cat fish, Hemibagrus nemurus Valenciennes 1840. Current Research Journal of Biological Sciences, 4: 242-246.

Moller, A.P. 2006. Parasitism and the regulation oh host populations. In: Thomas F.; Renaud, F.; Guégan, J.F. Parasitism and ecosystems. Oxford University Press, New York, p. 4-53.

Morais, A.M.; Varella, A.M.B.; Villacorta-Correa, M.A.; Malta J.C.O. 2009. A fauna de parasitos em juvenis de tambaqui Colossoma macropomum (Cuvier, 1818) (Characidae: Serrasalminae) criados em tanques-rede em lago de várzea da Amazônia central. Biologia Geral Experimental, 9: 14- 23.

Pinheiro, M.H.P.; Silva, J.W.; Nobre, M.I.S.; Pinheiro F.A. 1991. Cultivo de híbridos tambaqui, Colossoma macropomum Cuvier, 1818, com a pirapitinga, Piaractus brachypomum CUVER, 1818, na densidade de 5.000 peixes/ha. Revista Ciência Agronômica, 22: 77-87. 
Pojmanska, T. 1994. Infection of common carp and three introduced herbivorous fish from Zabieniec fish farm, in relation to their sizes. Acta Parasitologica, 39: 16-24.

Rohde, K.; Hayward, C.; Heap, M. 1995. Aspects of the ecology of metazoan ectoparasites of marine fishes. Journal Parasitological, 25: $945-970$.

Rózsa, L.; Reiczigel, J.; Majoros, G. 2000. Quantifying parasites in samples of hosts. The Journal of Parasitology, 86: 228- 232.

Santos, E.F.; Tavares-Dias, M.; Pinheiro, D.A.; Neves L.R.; Marinho, R.G.B.; Dias, M.K.R. 2013. Fauna parasitária de tambaqui Colossoma macropomum (Characidae) cultivado em tanque-rede no estado do Amapá, Amazônia oriental. Acta Amazonica, 43: 107-114.

Silva-Acuña, A.; Guevara, M. 2002. Evolución de las dietas comerciales sobre el crescimento de híbrido de Colossoma macropomum $x$ Piaractus brachypomus. Zootecnia Tropical, 20: 449 - 459.

Silva, R.M.; Tavares-Dias, M.; Dias, M.W.R.; Dias, M.K.R.; Marinho, R.G.B. 2013. Parasitic fauna in hybrid tambacu from fish farms. Pesquisa Agropecuária Brasileira, 48: 1049-1057.
Thatcher, V.E. 2006. Amazon fish parasites. $2^{\text {th }}$ ed. Pensoft Publishers, Sofia, Moscow, 508p.

Tavares-Dias, M.; Moraes, F.R.; Kronka, S. N. 2001a. Fauna parasitária de peixes oriundos de "pesque-pague" do município de Franca, estado de São, Brasil. II. Metazoários. Revista Brasileira de Zoologia, 18: 81-95.

Tavares-Dias, M.; Martins, M.L.; Moraes, F.R. 2001b. Fauna parasitária de peixes oriundos de "pesque-pague" do município de Franca, estado de São, Brasil. II. Protozoários. Revista Brasileira de Zoologia, 18: 67 - 79.

Tavares-Dias, M. 2011. Piscicultura continental no estado do Amapá: diagnóstico e perspectivas. Boletim de Pesquisa e Desenvolvimento 81, Embrapa Amapá, 42p.

Zar, J.H. Biostatistical analysis. 1999. $4^{\text {th }}$ d, New Jersey, PrenticeHall, 944p.

Recebido em 09/03/2014

Aceito em 19/09/2014 